Journal of Hyperbolic Differential Equations

Vol. 3, No. 4 (2006) 781-782

(C) World Scientific Publishing Company

\title{
AUTHOR INDEX (Volume 3)
}

Aguilar, G., Lévi, L. \&

Madaune-Tort, M.,

Coupling of

multidimensional

parabolic and hyperbolic

equations

Allen, P., Andersson, L. \&

Isenberg, J., Timelike

minimal submanifolds of general co-dimension in

Minkowski space time

Amadori, D. \& Serre, D., Asymptotic behavior of solutions to conservation laws with periodic forcing

Andersson, L., see Allen, P.

Antman, S.S., A priori bounds on spatial motions of incompressible nonlinearly elastic rods

Baranger, C. \&

Desvillettes, L., Coupling

Euler and Vlasov

equations in the context

of sprays: The

local-in-time, classical solutions

Bouchut, F. \& Frid, H., Finite difference schemes with cross derivatives correctors for multidimensional parabolic systems

Čanić, S., see Jegdić, K.

Chruściel, P.T. \& Łȩski, Sz., Polyhomogeneous solutions of nonlinear wave equations without corner conditions

Coulombel, J.-F. \&

Goudon, T.,
Entropy-based moment closure for kinetic equations: Riemann problem and invariant

1 (2006) $53 \quad$ Dafermos, C.M., Hyperbolic

4 (2006) 691

2 (2006) 387

$4(2006) 691$

3 (2006) 481

$1(2006) 1$

1 (2006) 27

3 (2006) 443

$1(2006) 81$ regions systems of balance laws with weak dissipation

Dafermos, M., A note on the collapse of small data self-gravitating massless collisionless matter

4 (2006) 649

3 (2006) 505

4 (2006) 589

Desvillettes, L., see Baranger, C.

$1(2006) 1$

Donatelli, D. \& Marcati, P., A dispersive approach to the artificial compressibility approximations of the Navier-Stokes equations in $3 D$

3 (2006) 575

Feldman, M., Ha, S.-Y. \& Slemrod, M., Self-similar isothermal irrotational motion for the Euler, Euler-Poisson systems and the formation of the plasma sheath

2 (2006) 233

Frid, H., see Bouchut, F.

Godin, P., Global centered waves and contact discontinuities of the axisymmetric Euler equations

1 (2006) 143

Goudon, T., see Coulombel, J.-F.

4 (2006) 649

Gues, O. \& Rauch, J., Nonlinear asymptotics for hyperbolic internal waves of small width

Ha, S.-Y., see Feldman, M.

Howard, P., Raoofi, M. \&

2 (2006) 233 pointwise bounds for 
perturbed viscous shock waves

Hunter, J.K., Short-time existence for scale-invariant

Hamiltonian waves

Isenberg, J., see Allen, P.

Jegdić, K., Keyfitz, B.L. \& Canić, S., Transonic regular reflection for the nonlinear wave system

Jin, S. \& Liao, X., A Hamiltonianpreserving scheme for high frequency elastic waves in heterogeneous media

Kagei, Y. \& Kawashima, S., Local solvability of an initial boundary value problem for a quasilinear hyperbolic-parabolic system

Kawashima, S., see Kagei, Y.

Keyfitz, B.L., see Jegdić, K.

Lagoutière, F., Large time behavior of numerical solutions of scalar conservation laws

LeFloch, P.G., Haar method, averaged matrix, wave cancellations, and $L^{1}$ stability for hyperbolic systems

Łęski, Sz., see Chruściel, P.T.

Lévi, L., see Aguilar, G.

Liao, X., see Jin, S.

Madaune-Tort, M., see Aguilar, G.

Marcati, P., see Donatelli, D.

Mazzucato, A.L. \& Nistor, V., Mapping properties of heat kernels, maximal regularity, and semi-linear parabolic equations on noncompact manifolds

\author{
2 (2006) 297 \\ Morawetz, C. S. \& \\ Thomases, B., A decay \\ theorem for some \\ symmetric hyperbolic \\ systems
}

2 (2006) 247

4 (2006) 691

3 (2006) 443

4 (2006) 741

2 (2006) 195

2 (2006) 195

3 (2006) 443

4 (2006) 631

4 (2006) 701

1 (2006) 81

1 (2006) 53

4 (2006) 741

1 (2006) 53

3 (2006) 575

4 (2006) 599 A.L. B., Local energy decay for solutions of multi-dimensional isotropic symmetric hyperbolic systems M.

Smoller, J. \& Temple, B., resolve the flatness problem Entropy stable approximations of with no artificial numerical viscosity

Thomases, B., see Morawetz, C. S. T.C. H.J., Convergence rate for the compressible with external force

Xin, Z. \& Yuan, H., Vacuum state for spherically symmetric solutions of the compressible Navier-Stokes equations

Yang, T., see Ukai, S.

Yuan, H., see Xin, Z.

Zhao, H. J., see Ukai, S.

P.
3 (2006) 475

Nistor, V., see Mazzucato,

4 (2006) 599

2 (2006) 297

2 (2006) 269

2 (2006) 387

Serre, D., see Amadori, D.

Sideris, T.C. \& Thomases,

Slemrod, M., see Feldman,

4 (2006) 673

2 (2006) 233 How inflation is used to

Tadmor, E. \& Zhong, W., Navier-Stokes equations

3 (2006) 529

2 (2006) 375

Temple, B., see Smoller, J.

3 (2006) 475

Thomases, B., see Sideris,

4 (2006) 673

Ukai, S., Yang, T. \& Zhao, Navier-Stokes equations

3 (2006) 561

Zhong, W., see Tadmor, E. Zumbrun, K., see Howard,

3 (2006) 403

3 (2006) 561

3 (2006) 403

3 (2006) 561

3 (2006) 529

2 (2006) 297 